\title{
1 Graphical Table of Contents
}

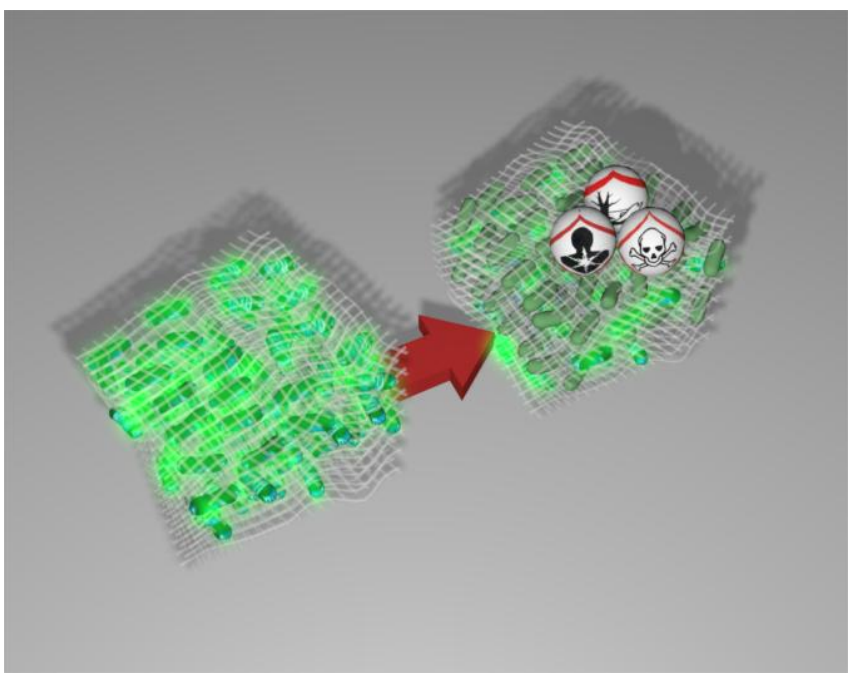

3 A composite material based on bacterial nanocellulose operating as both a culture scaffold and a

4 biosensing substrate and luminescent bacteria Alivibrio fischeri operating as a bio-indicator is

5 reported. This nanobiocomposite is utilized as a simple-to-fabricate and user-friendly device for

6 toxicity detection via determination of the bioluminescent inhibition caused by the exposure to

7 various contaminants.

9 Bioluminescent Nanopaper for the Fast Screening of Toxic Substances

10 Jie Liu, Eden Morales-Narváez, Jahir Orozco, Teresa Vicent, Guohua Zhong*, Arben Merkoçi* 


\section{Bioluminescent Nanopaper for the Fast Screening of}

\section{Toxic Substances}

Jie Liu ${ }^{1,2}$, Eden Morales-Narváez ${ }^{1}$, Jahir Orozco ${ }^{1}$, Teresa Vicent ${ }^{3}$, Guo-Hua Zhong ${ }^{2 *}$ and

$18{ }^{1}$ Catalan Institute of Nanoscience and Nanotechnology (ICN2), CSIC and the Barcelona Institute

19 of Science and Technology, Campus UAB, Bellaterra, 08193 Barcelona, Spain

$20 \quad{ }^{2}$ Laboratory of Insect Toxicology, Key Laboratory of Pesticide and Chemical Biology, Ministry

21 of Education, South China Agricultural University, Guangzhou 510642, P. R. China

$22{ }^{3}$ Departament d'Enginyeria Química, UniversitatAutònoma de Barcelona, Bellaterra Barcelona

23 08193, Spain

$24{ }^{4}$ Institucio Catalana de Recerca i Estudis Avançats (ICREA), Barcelona 08010, Spain

27 *guohuazhong@scau.edu.cn

28 *arben.merkoci@icn2.cat 
31 Abstract: Environmental pollution is threatening human health and ecosystems as a result of

32 modern agriculture techniques and industrial progress. A simple nanopaper-based platform

33 coupled with luminescent bacteria Alivibrio fischeri (A. fischeri) as a bio-indicator is presented

34 for the rapid and sensitive toxicity evaluation of contaminants. When exposing to toxicants, the

35 light inhibition on A. fischeri-decorated bioluminescent nanopaper (BLN) can be quantified and 36 analyzed to classify the toxic level of a pollutant. The BLN composite was characterized in terms

37 of morphology and functionality. Given the outstanding biocompatibility of nanocellulose in

38 bacteria proliferation, BLN achieved high sensitivity with low cost and simplified procedure

39 compared to a conventional instrument for lab use only. The broad applicability of BLN devices

40 upon environmental samples was studied in spiked real matrix (lake and sea water), and their

41 potential for the direct and in-situ toxicity screening was demonstrated. The BLN architecture

42 can not only survive but also maintain its function during freezing storage and recycling process,

43 which endowed BLN system with competitive advantages as a deliverable, ready-to-use device

44 in large-scale manufacturing. The novel luminescent bacteria-immobilized nanocelullose-based

45 device shows outstanding capabilities for toxicity bioassay of hazardous compounds, bringing

46 new possibilities for cheap and efficient environmental monitoring of potential contamination.

47 Keywords: bacterial nanocellulose, nanopaper, Aliivibrio fischeri, bioluminescent device, 48 toxicity bioassay 


\section{Introduction}

54 Along with the great benefits from modern agricultural and industrial development, it has been

55 attracting more and more attention over the environmental pollution that originating from human

56 activities. Conventional techniques such as gas or liquid chromatography, dissolved oxygen

57 content and chemical oxygen demand have been widely applied to characterize toxic compounds

58 [1-5]. However, these techniques only indicate the nature (structure and composition) of the

59 pollutants, but their biological effect on live organisms is also important to investigate [6].

60 Therefore, a range of toxicity bioassays that involves various organisms (e.g., plants, aquatic

61 invertebrate, fish, algae and microorganisms) have been developed by taking advantages of the

62 biological response of live organisms against contaminants [7-11]. Particularly, many

63 microorganism species have been selected because of their ecological importance and

64 physiological diversity [8, 11]. In 1978, the commercially available, ready-to-use kit Microtox ${ }^{\circledR}$

65 assay was developed based on luminescent marine bacterium Aliivibrio fischeri (A. fischeri) as a

66 bio-indicator to determine the toxicity of environmental samples [6,9]. A. fischeri can emit

67 blue-green light during its metabolism, which could be inhibited when exposing to toxic

68 substances. By measuring the light inhibition, the toxicity can be converted as $\mathrm{EC}_{50}$, which

69 expresses the concentration of toxicant corresponding to a $50 \%$ inhibitory effect. So far,

70 Microtox $^{\circledR}$ test has shown broad sensitivity and applicability to more than 2,700 different

71 pollutants of interest [12-16].

72 Due to its high cost and unique analytical equipment, the Microtox ${ }^{\circledR}$ kit may lack the ability of

73 large-scale in-situ screening. An alternative based on immobilized bacteria may provide a

74 feasible scheme to perform low-cost and simple-to-operate detection of hazardous compounds as

75 well as maintain highly functional bacteria [17-20]. In this process, the material used as substrate

76 is crucial because of its dramatic effects on the survival and function of the immobilized bacteria. 
77 Recently, bacterial cellulose nanopaper produced by Acetobacter xylinum has shown up excellent

78 potential in several fields [21-23]. Thanks to its remarkable physical properties, special surface

79 chemistry and excellent biological properties (biocompatibility and biodegradability), bacterial

80 cellulose nanopaper has been selected as a culture skeleton for the cell proliferation [24-25]

81 which suggest its potential to be a desirable substrate for bioluminescent A. fischeri.

82 Herein, we present a cheap, sensitive, efficient and robust platform for toxicity bioassay based

83 on bioluminescent nanopaper (BLN) devices consisting of bacteria as a bio-indicator and

84 bacterial nanocellulose as bio-scaffold (see Scheme 1). While A. fischeri immobilized on

85 nanopaper is exposed to a toxicant, the bioluminescence is efficiently inhibited as a toxicity

86 indicator in a short time (5 to 15 minutes). Firstly, A. fischeri was immobilized into the fiber

87 networks of nanocellulose to form the BLN composite. The as-prepared nanopaper devices were

88 characterized in terms of their morphology and function. The fact of having luminescent bacteria

89 fully distributed in a cheap and green bio-substrate offers the possibility of a rapid toxicity

90 evaluation of a myriad of toxic compounds. Diuron, tributyltin (TBT) and polybrominated

91 diphenyl ether (PBDE) were chosen as typical contaminants that sensitively induced the

92 bioluminescence inhibition of BLN to varying degrees. The sensitivity and applicability of BLN

93 upon real matrix were also determined. Through frozen storage and recycling process, the BLN

94 architectures exhibited competitive advantages as robust, deliverable and ready-to-use devices.

95 The BLN-based bioassay demonstrated outstanding capabilities for toxicity evaluation with a

96 miniaturized setup and flexible procedure, which brings innovative possibilities for general

97 toxicity screening and environmental monitoring.

98 1. Materials and methods

$99 \quad 1.1$ Reagents and equipment 
Bacterial cellulose nanopaper was purchased from Nanonovin Polymer Co. (Mazandaran, Iran).

101 Diuron (98\%), tributyltin chloride (96\%), acetonitrile, ethanol, sodium chloride, tryptone, sea

102 salts, yeast extract, sucrose, glycine and glycerol (99\%) were purchased from Sigma-Aldrich

103 (Taufkirchen, Germany). Stock solutions of diuron $\left(10 \mathrm{~g} \mathrm{~L}^{-1}\right)$ and TBT $(1 \mathrm{mM})$ were prepared in

104 acetonitrile and ethanol, respectively and stored at $4{ }^{\circ} \mathrm{C}$. Polybrominated diphenyl ether (PBDE)

$105\left(1 \mathrm{mg} \mathrm{L}^{-1}\right)$ was purchased from AccuStandard Inc (New Haven, CT, USA). Cellulose membrane

106 CFSP001700 was acquired from Millipore (Billerica, MA, USA). Scanning electron microscopy

107 (SEM) imaging was performed through a Magellan 400L SEM High Resolution SEM (FEI,

108 Hillsboro, OR, USA). Photoluminescence images were obtained using a Typhoon 9410 Variable

109 Mode Imager (GE, Freiburg, Germany). Confocal imaging was performed using a SP5 confocal

110 microscope (Leica, Wetzlar, Germany). Bioluminescence intensity was estimated using ImageJ

111 1.46r (Wayne Rasband, National Institutes of Health, Bethesda, MD, USA). The optical density

112 of bacteria was measured by Perkin Elmer Victor3 Multilabel Plate Counter (Waltham, MA,

113 USA). Microtox ${ }^{\circledR}$ assay was performed on a Microtox ${ }^{\circledR}$ M500 toxicity analyser (Modern Water,

114 New Castle, DE, USA). Lake water samples were collected from Sant Cugat Lake (Barcelona,

115 Spain). Seawater samples were extracted from Masnou Beach (Barcelona, Spain). Lake water

116 and seawater samples were filtered using filter paper and then a nitrocellulose membrane $(0.025$

$117 \mu \mathrm{m}$, Millipore, Billerica, Massachusetts, USA) prior to use.

$118 \quad$ 1.2 The cultivation of A. fischeri and its bioluminescence emission

119 A. fischeri was isolated from Microtox ${ }^{\circledR}$ reagent by aseptically adding $1 \mathrm{~mL}$ of reconstruction 120 solution to a microbial reagent vial and then inoculating the homogenized solution on a marine 121 agar plate. After $24 \mathrm{~h}$, the sole colony emitting bioluminescence was picked up by sterilized loop 122 and inoculated into $50 \mathrm{~mL}$ of marine broth in a $250-\mathrm{mL}$ Erlenmeyer flask placed on an 
123 incubation shaker at $25^{\circ} \mathrm{C}, 140 \mathrm{rpm}$. The modified marine broth (MB) [26] contained tryptone (5

$\left.124 \mathrm{~g} \mathrm{~L}^{-1}\right)$, yeast extract $\left(3 \mathrm{~g} \mathrm{~L}^{-1}\right)$, glycerol $\left(3 \mathrm{~mL} \mathrm{~L}^{-1}\right)$ and sea salts $\left(40 \mathrm{~g} \mathrm{~L}^{-1}\right)$. To prepare the solid

125 marine agar (MA) plate, $15 \mathrm{~g} \mathrm{~L}^{-1}$ of agar powder was added in marine broth. Both media were

126 sterilized at $121{ }^{\circ} \mathrm{C}$ for $20 \mathrm{~min}$ and cooled/fused to room temperature prior to use.

127 A. fischeri was respectively inoculated to three substrates: MA, MB and sterile nanocellulose

128 pieces (5mm of diameter, round-shaped pieces). To prepare sufficient nutrient for bacteria

129 multiplication, nanocellulose was previously immersed in marine broth for nutrient adsorption

130 for $2 \mathrm{~h} .1 \mu \mathrm{L}$ of bacteria suspension at 1.2 of optical density $\left(\mathrm{OD}_{600 \mathrm{~nm}}\right)$, which was around

$1312.3 \times 10^{8}$ of colony-forming units (CFU) $\mathrm{mL}^{-1}$, was inoculated in $100 \mu \mathrm{L}$ of marine broth/agar or

132 one piece of nanopaper in the individual well of 96-well microplate. 9 replicates and three blanks

133 were settled for each substrate. The $\mathrm{OD}_{600 \mathrm{~nm}}$ and luminescent intensity were respectively

134 measured through a microplate reader and a Typhoon 9410 scanner every $2 \mathrm{~h}$ within $24 \mathrm{~h}$ to

135 determine the growth and bioluminescence tendency among three groups.

136 Additionally, the procedure for routine cultivation was performed as follows. $50 \mu \mathrm{L}$ of

137 bacterial suspension at 1.2 of $\mathrm{OD}_{600 \mathrm{~nm}}\left(2.3 \times 10^{8} \mathrm{CFU} \mathrm{mL}^{-1}\right)$ was inoculated in $50 \mathrm{~mL}$ of $\mathrm{MB}$ in a

138 250-mL Erlenmeyer flask and incubated on a shaker at $25^{\circ} \mathrm{C}, 140 \mathrm{rpm}$.

$139 \quad 1.3$ The preparation of BLN and its morphological observation

140 In order to obtain BLN samples emitting homogeneous bioluminescence, the sterilized 141 nanocellulose pieces were added into $50 \mathrm{~mL}$ of $\mathrm{MB}$ in a $250-\mathrm{mL}$ Erlenmeyer flask with $0.1 \%$ of 142 inoculum. After $18 \mathrm{~h}$ of incubation, the BLN pieces were individually placed into the wells of 143 96-well microplate prior to observation. The appearances of bare nanopaper and BLN were 144 separately captured by iPhone 6.0 (Apple Inc, Cupertino, CA, USA) and Typhoon 9410 scanner 
145 to analyze their physical and luminescent images. The composites ready for SEM imaging were 146 prepared according to a published method without final sputtering of gold [27]. Briefly, bare 147 nanopaper and BLN pieces were dehydrated by gradient elution using ethanol and 148 hexamethyldisilazane (HMDS) for the critical point drying. As for the confocal microscopy, the 149 BLN composites were stained by the mixture of $4 \mu \mathrm{L}$ of Hoechst 33342 (Molecular Probe Inc, 150 Eugene, OR, USA) and $500 \mu \mathrm{L}$ of phosphate-buffered saline for 15 min prior to imaging.

$151 \quad 1.4$ Toxicity assay using Microtox ${ }^{\circledR}$

152 Microtox $^{\circledR}$ reagents were supplied by Modern Water (New Castle, DE, USA) as freeze-dried 153 powder batches and stored at $-20^{\circ} \mathrm{C}$ prior to use. The toxicity analysis of three contaminants, 154 diuron $\left(100 \mathrm{mg} \mathrm{L}^{-1}\right)$, TBT $(0.1 \mathrm{mM})$ and $\operatorname{PBDE}\left(1 \mathrm{mg} \mathrm{L}^{-1}\right)$, were conducted respectively 155 according to Microtox ${ }^{\circledR}$ protocol (AZUR Environmental, New Castle, DE, 1998). Briefly, the 156 serial dilutions of the target compound were individually mixed with the same volume of 157 reconstructed reagents. The inhibition of bioluminescence was measured by M500 luminescent 158 analyser after exposure 5 and 15 min and expressed as the $\mathrm{EC}_{50}$ values.

$159 \quad$ 1.5 Toxicity assay using bioluminescent bacterial suspension

160 After $18 \mathrm{~h}$ of routine cultivation, A. fischeri was collected by centrifugation at $6000 \mathrm{rpm}$ for 10 161 min, washed twice and suspended in $10 \mathrm{~mL}$ of $2 \% \mathrm{NaCl} .100 \mu \mathrm{L}$ of bacterial suspension was 162 first added by a multichannel pipette to each well of a 96-well microplate. 1:2 serial dilutions of 163 diuron, TBT and PBDE were performed by transferring $1 \mathrm{~mL}$ sample into $1 \mathrm{~mL}$ of $2 \% \mathrm{NaCl}$ and 164 mixed after each transfer. $100 \mu \mathrm{L}$ of each diuron/PBDE (or $50 \mu \mathrm{L}$ of TBT) dilutions were added 165 into the well containing $100 \mu \mathrm{L}$ of bacterial suspension and mixed. Three replications were 166 prepared and the treatments without toxic compounds were carried out as control. After exposure 
167 for 5 and $15 \mathrm{~min}$, the bioluminescence intensity in microplates was scanned by a Typhoon 9410

168 scanner and analyzed through ImageJ 1.46 r.

$169 \quad$ 1.6 Toxicity assay u sing bacteria-decorated BLN

170 The BLN pieces with homogeneous luminescence were prepared as described previously.

171 After $18 \mathrm{~h}$ of cultivation, bacteria-decorated BLN composites were collected and placed

172 individually in each well of a 96-well microplate. 1:2 serial dilutions of diuron, TBT and PBDE

173 were performed as described. Each BLN piece was mixed with $100 \mu \mathrm{L}$ of each diuron/PBDE (or

$17450 \mu \mathrm{L}$ of TBT) dilutions. The mixtures without toxic compounds were carried out as blank

175 controls. After exposing 5 and $15 \mathrm{~min}$, the bioluminescence intensity in microplates was scanned

176 by a Typhoon 9410 scanner and analyzed via ImageJ $1.46 \mathrm{r}$.

$177 \quad 1.7$ Toxicity assay in real matrixes

178 To explore the applicability of BLN devices upon real matrix, toxicity bioassay using BLN

179 was performed in both lake water and seawater systems. Likewise, the evaluation by bacterial

180 suspension was designed as a comparison group. The stock solutions of diuron, TBT and PBDE

181 were blended in lake/sea water to reach working concentrations. 1:2 serial dilutions of target

182 compounds were prepared by transferring $1 \mathrm{~mL}$ sample into $1 \mathrm{~mL}$ of lake/sea water and mixing

183 after each transfer. Then the bioassay using bacterial suspension or BLN pieces were conducted

184 as described before. The bioluminescent images were collected by a Typhoon 9410 scanner and

185 analyzed using ImageJ 1.46 r.

$186 \quad 1.8$ Frozen-thawed process 
187 An easy-to-operate frozen-thawed strategy was adopted to realize the storage of BLN platform.

188 Basically, the biomaterials are frozen under the protection of reagents such as polyalcohols, 189 amino acids and disaccharides and thawed to normal status prior to use. The recovered

190 biomaterials are expected to maintain viable or normal function after a frozen-thawed process. In

191 this case, three general protective agents (sucrose, glycine and glycerol) were employed to select

192 the most suitable protectant for the frozen-thawed process of BLN. Specifically, each protectant 193 was prepared as $1 \%, 3 \%, 5 \%, 10 \%$ and $15 \%$ solutions with Milli-Q water and sterilized at

$194121{ }^{\circ} \mathrm{C}$ for $20 \mathrm{~min}$. BLN pieces were placed individually in a 96 -well microplate and the 195 luminescent intensity was scanned and recorded as the initial intensity. $50 \mu \mathrm{L}$ of protectant 196 solution was mixed with single BLN piece and three replicates were performed in each 197 concentration. The bioluminescence intensity was recorded as "adding protectant" before 198 freezing at $-20{ }^{\circ} \mathrm{C}$. After freezing for $2 \mathrm{~h}$, the composites were thawed and the luminescence 199 at "just-thawed" stage was scanned for further analysis. Inside each well, the protectant liquid 200 was removed and then $50 \mu \mathrm{L}$ of $\mathrm{MB}$ solution was added for incubation at $25^{\circ} \mathrm{C}, 30 \mathrm{~min}$. Finally, 201 the bioluminescence of recovered BLN pieces was scanned to evaluate the frozen-thawed 202 process.

$203 \quad 1.9$ The reuse of BLN

204 To investigate the robustness of the BLN device, a set of 6 sterile nanocellulose pieces were 205 added into $25 \mathrm{~mL}$ of $\mathrm{MB}$ culture inoculated with $0.1 \%$ of inoculum at $25{ }^{\circ} \mathrm{C}, 140 \mathrm{rpm}$. After 206 cultivation of $18 \mathrm{~h}, \mathrm{BLN}$ were scanned to measure the luminescence as the initial intensity. Then 207 those $\mathrm{BLN}$ pieces were washed twice by $2 \% \mathrm{NaCl}$ and sterilized at $121{ }^{\circ} \mathrm{C}$ for $20 \mathrm{~min}$. The 208 process was repeated by inoculating $25 \mu \mathrm{L}$ of bacteria into $25 \mathrm{~mL}$ MB culture containing those 
209 cooled nanopaper pieces and cultivating as a routine set. At the end of each cycle, the

210 bioluminescence intensity was scanned and compared with the initial one for 10 cycles.

$211 \quad 1.10$ Data analysis

212 ImageJ 1.46r software was employed to analyze the bioassay pictures scanned by Typhoon.

213 First, the scanning pictures were adjusted for better contrast by "Image" option (this process does

214 not modify the original grayscale values of the images). To distinguish the bioassays from

215 different contaminants, the color of each treatment was changed in "Image" and "Lookup Tables"

216 option. Then the bioluminescence intensity was measured by "Oval selection" tool and calculated

217 in grayscale.

218 To determine the $\mathrm{EC}_{50}$ of each toxicant, the observed concentration-response data were fitted

219 to the modified non-linear equation (1) [28]:

$220 I=\alpha+(\beta-\alpha) /\left(1+10^{\left(\left(\log E C_{50}-\log C\right) * k\right)}\right)$

221 where $I$ is the luminescent intensity; $\alpha$ and $\beta$ are the parameters of the models; $\mathrm{EC}_{50}$ is the

222 concentration of test chemicals that provokes a response half way between the maximal $(\beta)$

223 response and the maximally inhibited $(\alpha)$ response; $C$ represents the test concentration of

224 chemicals and $k$ describes the steepness of the curve.

\section{2. Results and Discussion}

226 In marine ecosystem, the bioluminescence of $A$. fischeri was the outcome of the cell-to-cell

227 communication. Once the bacteria reach to a high cell density, they switch on the "quorum

228 sensing" mode and emit light [29-30]. In order to understand its growth cycle and luminescent

229 emission, A. fischeri was inoculated and cultivated in liquid and agar cultures, respectively.

230 Within $20 \mathrm{~h}$, bacteria experienced the lag, exponential and stationary phases sequentially (Figure 
231 1D). At 12 to $14 \mathrm{~h}$, the bioluminescence in two cultures peaked at the highest intensity but 232 decreased sharply afterwards, implying the instability of light emission in both systems (Figure 233 1E). Then $A$. fischeri at $2.3 \times 10^{8} \mathrm{CFU} \mathrm{mL}^{-1}$ (calibrated in Figure S1) was inoculated on the 234 round-shaped pieces of nanocellulose (5 $\mathrm{mm}$ of diameter) and underwent three physiological 235 phases as normal (Figure 1D). Unlike the dramatic fluctuations in conventional cultures, the 236 bioluminescence in BLN was more stable and persistent because of the biocompatible and 237 flexible bio-support (Figure 1E). In smart phone pictures, the colour of BLN turned yellowish as 238 its turbidity was higher than the transparent bare nanocellulose (see Figure 1A and 1B, left). By 239 scanning, BLN sample showed intensive and homogenous luminescence emission (see Figure 1B, 240 right). Meanwhile, the SEM images displayed that the network of nanocellulose remained as rich 241 and crossed nets after immobilization but densely filled with bacteria (see Figure 1a and 1b).

242 During the bacteria immobilization process, the quantity of bacteria was increased due to the

243 proliferation of numerous cells. Some newly divided bacteria were trapped into the nanocellulose

244 network. With their growing, the size of bacteria was enlarged in the life cycle, which spread the

245 surrounding nanocellulose fibres and made the network pores bigger than those without bacteria.

246 For example, in Fig.1 b, the background nanocellulose (without bacteria) demonstrated the same

247 density as Fig.1 a (the bare nanopaper). Therefore, the difference in density showing in before-

248 and after-immobilization process (Figure 1a and $1 \mathrm{~b}$ ) would be caused by the stretch of growing

249 bacteria. Besides, this phenomenon also demonstrated the excellent flexibility of nanocelullose

250 paper. Surprisingly, one bacterium was even undergoing the cell division process, proving the 251 impressive biocompatibility of nanocellulose in A. fischeri's metabolism (Figure S2D). 252 Moreover, the three-dimensional (3D) confocal microscopy images of BLN illustrated the 
253 intensive and homogenous distribution of A. fischeri onto/into the nanopaper structure, which in

254 fact contributed to the strong light emission of BLN composite (Figure 1C, 1c and Movie S1).

255 Typically, suspension/adherent culture is used for cell enrichment that can efficiently provide a

256 large number of cells. Based on new culture substrates and techniques, the 3D formation of cell

257 communities has become a hot topic recently. Bacterial nanocellulose secreted by Acetobacter

258 xylinum has showed up as one of those emerging materials for cell/tissue engineering of stem

259 cells [24, 31], tumor [25] and cartilage [32-33]. As we first report here, bacterial nanocellulose

260 has exhibited its outstanding biocompatibility with marine bacteria $A$. fischeri, which realized

261 normal proliferation of bacteria on an abundant, low-cost substrate with stable genetic expression.

262 Besides, the use of general luminescent equipment enabled a more rapid and simultaneous

263 measurement of bioluminescence, leading to a bio-composite that operates as a simple-to-

264 fabricate and user-friendly device in practical applications such as toxicity determination.

265 Environmental monitoring is a prevalent topic in the scientific field and the public areas, in

266 which toxicity bioassays have been a widely-used mean for their significant importance to

267 determine the biological impacts of known/unknown pollutants on living organisms.

268 Commercially, Microtox ${ }^{\circledR}$ has been recommended as a standard tool to reveal the toxic level of

269 sole/complex toxin(s) towards aquatic environment in the last decades [6]. In this study, the

270 toxicity levels of diuron, TBT and PBDE was classified by Microtox ${ }^{\circledR}$ (Table S1, Figure S3),

271 showing that $A$. fischeri was highly sensitive to these three xenobiotics. In Microtox ${ }^{\circledR}$ kit, the

272 bio-reagent $A$. fischeri is prepared as a freeze-dried powder with high uniformity for toxicity

273 evaluation and typically stored at low temperature $\left(-20{ }^{\circ} \mathrm{C}\right)$. Since its high price and unique

274 measurement, the kit may lack the ability of large-scale screening in practice. Nevertheless,

275 using immobilized bacteria may facilitate a low-cost and simple-to-fabricate bioassay platform. 
276 Hence, we investigated the toxicity assays of three compounds via A. fischeri-decorated BLN. As

277 displayed in Figure 2, the optical intensity of each treatment decreased gradually with the

278 decrease of toxic concentrations, which fitted well in the symmetrical sigmoidal curves (see also

279 Figure S5). By calculation, the $\mathrm{EC}_{50}$ of diuron, TBT and PBDE were $108.2 \mathrm{mg} \mathrm{L}^{-1}, 0.24 \mathrm{mg} \mathrm{L}^{-1}$

280 and $8.7 \mu \mathrm{g} \mathrm{L}^{-1}$, respectively. Comparing to those results from Microtox ${ }^{\circledR}$ and free-cell evaluation

281 (Figure S3 and S4), the use of BLN showed comparable analytical behavior and accuracy (Table

$282 \mathrm{~S} 1)$. To be specific, higher $\mathrm{EC}_{50}$ values were obtained from free-cell tests $\left(123 \mathrm{mg} \mathrm{L}^{-1}, 0.46 \mathrm{mg}\right.$

$283 \mathrm{~L}^{-1}$ and $11.6 \mu \mathrm{g} \mathrm{L}^{-1}$, respectively), which indicated that bacteria-immobilized BLN devices were

284 more sensitive than free-cell suspension. In comparison to standard Microtox ${ }^{\circledR}$ tests, similar

285 performance was obtained as the $\mathrm{EC}_{50}$ of TBT and PBDE were $0.27 \mathrm{mg} \mathrm{L}^{-1}$ and $15.8 \mu \mathrm{g} \mathrm{L}^{-1}$ using

286 Microtox $^{\circledR}$ (that was $0.24 \mathrm{mg} \mathrm{L}^{-1}$ and $8.7 \mu \mathrm{g} \mathrm{L}^{-1}$ via BLN). Different from TBT and PBDE tests,

287 less sensitive performance was observed in diuron as the $\mathrm{EC}_{50}$ was $21.1 \mathrm{mg} \mathrm{L}^{-1}$ by Microtox ${ }^{\circledR}$

288 whilst $108.2 \mathrm{mg} \mathrm{L}^{-1}$ via BLN. According to reported studies, however, the $\mathrm{EC}_{50}$ value of diuron

289 varied significantly from 8 to $86 \mathrm{mg} \mathrm{L}^{-1}$ (Table S2), which might imply that different batches of

290 bio-reagent $A$. fischeri or operation conditions might have an effect on the bioassay sensitivity,

291 leading to the distinct toxicity levels of diuron. In the present work, the luminescence inhibition

292 induced by diuron in BLN could be fitted in the statistic model with predictable $\mathrm{EC}_{50}$ level,

293 which confirmed its possibility for further study. As expected, A. fischeri retained its sensitivity

294 to xenobiotics in the form of BLN devices. Taking the advantage of the simple fabrication and

295 easy operation, the proposed nanobiocomposite facilitates a rapid, sensitive, non-invasive and

296 broadly available platform for the evaluation of various toxicants.

297 The applicability to real samples is regarded as the ultimate goal for a bioassay platform. In

298 this context, simplification of the test process and avoiding secondary pollution coming from the 
299 test itself is highly desired. The proposed BLN device was expected to function with

300 environmental samples so as to demonstrate its accessibility for practical applications.

301 Consequently, we estimated its sensitivity in both lake water and seawater, whose no adverse

302 effect on BLN luminescence properties was previously confirmed (see Figure S6 and S7). The

303 inhibition of bacterial luminescence by exposing to toxic compounds for 5 and 15 min was

304 measured and plotted in Figure 3. In real matrixes, BLN devices kept stable and higher

305 sensitivity towards TBT in spiked lake water or sea water. Comparing the $\mathrm{EC}_{50}$ to those samples

306 analyzed in pure water, the $\mathrm{EC}_{50}$ of TBT was $0.27 \mathrm{mg} \mathrm{L}^{-1}$ in lake water and even lower at 0.18

$307 \mathrm{mg} \mathrm{L}^{-1}$ in spiked sea water (that was $0.24 \mathrm{mg} \mathrm{L}^{-1}$ in pure water test). However, the sensitivity of

308 BLN composites decreased towards diuron and PBDE (Table S3). Particularly, the $\mathrm{EC}_{50}$ of

309 PBDE increased dramatically to approximately $30 \mu \mathrm{g} \mathrm{L}{ }^{-1}$ in spiked real matrixes compared to 8.7

$310 \mu \mathrm{g} \mathrm{L}^{-1}$ in pure water. The matrix effect could possibly be a major reason for the lower sensitivity

311 of BNL devices in real samples. Bacteria A. fischeri was discovered and isolated from a marine

312 creature. In the laboratory incubation, bacteria cells also require the high salinity culture to

313 proliferate and emit bioluminescence. In the real matrix, especially the sea water, the matrix may

314 contain complex ingredients or trace elements that provided A. fischeri a suitable environment as

315 buffer, which could enhance its resistance against toxic xenobiotics, leading to lower sensitivity.

316 In addition, the toxic agents at low concentrations demonstrate stimulatory effects to living

317 organisms, which was generally observed and described as hormesis effect [34-35]. Since the

318 low concentration of PBDE (at $0.2 \mathrm{mg} \mathrm{L}^{-1}$ ) was spiked in lake and sea water, the less sensitive

319 performance of A. fischeri-decorated BLN in real matrix could be a result of the low-dose

320 stimulation relationship phenomenon. 
321 According to these results, BLN platform exhibited many advantages in the application of 322 toxicity bioassay. First, the BLN device was simple to fabricate based on cheap and abundant 323 ingredients. Bacteria-derived nanocellulose as cell culture skeleton was a sustainable biomaterial 324 with high permeability, flexibility and great biocompatibility to luminescent marine bacteria $A$. 325 fischeri. These inherent properties of nanocellulose enabled BLN to be a low-cost, controllable 326 and easy-to-assembly bio-composite representing no threat to the environment. After bacteria 327 immobilization, the biological character of $A$. fischeri was retained in the BLN architecture, 328 keeping its stable and persistent bioluminescence emission in toxicity screening. Second, 329 sensitivity is the primary rule for toxicity screening. BLN platform demonstrated a high 330 sensitivity towards hazardous compounds that is comparable with the gold standard of this kind 331 of bioassays. More importantly, BLN demonstrated its broad applicability with real matrix, 332 which highlighted its potential for the practical in-situ use with shortened and simplified process.

333 In addition, the bioluminescence intensity of BLN setup could be measured by a one-step 334 simultaneous scan with a general scanner or a microplate reader instead of a special 335 luminescence analyzer. No particular expertise or skills is required during the bioassay process, 336 which allowed the wide use of BLN platform for practical needs. Overall, the fabrication of BLN 337 based on nanocellulose as a bio-support and A. fischeri as a bio-indicator may facilitate a rapid, 338 sensitive, low-cost, controllable and non-invasive toxicity bioassay with general and portable 339 equipment. Since A. fischeri exhibited its broad sensitivity to thousands of chemicals, BLN 340 device is amenable to being applied as a broadly accessible platform for toxicity screening of 341 xenobiotic complexes under in-situ conditions.

342 Storability and reusability may endow the present BLN platform with more competitive 343 advantages for the further large-scale fabrication and commercial application. Consequently, we 
344 also investigated the performance of BLN after frozen-thawed process and recycling process,

345 respectively. In general, the frozen-thawed process was conducted under the protection of 346 cryoprotective agents including polyalcohol, amino acids and disaccharides [36-38]. As showing

347 in Figure 4, we investigated the recovered bioluminescence of BLN devices frozen with 348 glycine/sucrose/glycerol at $-20{ }^{\circ} \mathrm{C}$. Due to the potential toxicity of cryoprotectants, the 349 bioluminescence of BLN slightly decreased after adding those protective agents. The group 350 treated with glycine indicated the best preservation of bioluminescence since more than $60 \%$ of 351 the bioluminescent intensity regained by applying 5\%, 10\% and $15 \%$ of glycine. Particularly, the

352 luminescence of BLN devices under the protection of 5\% of glycine resumed to approximate $80 \%$ 353 of the initial intensity, even surpassing the luminescent level before freezing, which suggested 354 that BLN could tolerate the freezing storage with $5 \%$ of glycine. Thus, we employed those 355 frozen-thawed BLN devices (in 5\% of glycine) to perform a toxicity assay using diuron as a 356 model pollutant. In Figure 4D, the frozen-thawed BLN could estimate the toxic level of diuron

357 ( $\mathrm{EC}_{50}$ at $195.6 \mathrm{mg} \mathrm{L}^{-1}$ ) (that is $108.2 \mathrm{mg} \mathrm{L}^{-1}$ compared to that from the unfrozen platform).

358 Although the frozen-thawed device showed lower sensitivity than unfrozen platform, it still

359 indicated that BLN composites had the potential to remain the bio-function in toxicity bioassay

360 through cryopreservation process. In a future study, optimization on freezing process would be

361 required to improve the survival of $A$. fischeri and the sensitivity of frozen BLN devices, for

362 instance, using lyophilisation technique.

363 Next, we evaluated the reusability of BLN devices by recycling used BLN and repeating the 364 bacteria immobilization process. Figure 4E illustrates the stable intensity of BLN luminescence 365 after 10 cycles, implying the great robustness of BLN composites and their biocompatibility to 366 bacteria $A$. fischeri was retained after recycling. Taking the benefit of its reusability, these robust 
367 BLN devices not only realized a green and low-cost fabrication, but also might have great

368 potential for an automatically assembling setup, like wiping and reloading luminescent bacteria

369 after use. Herein, the BLN device presented its properties as a novel, robust and green platform

370 for toxicity assays, highlighting its unique potential in large-scale fabrication and ready-to-use

371 application by frozen-thawed preservation.

\section{3. Conclusions}

373 We have demonstrated a low-cost, green, controllable and simple-to-fabricate BLN device

374 based on bacterial nanocellulose as both a culture scaffold and biosensing substrate and

375 luminescent bacteria A. fischeri as a bio-indicator. By determining the luminescent inhibition

376 caused by the exposure of toxicants, the novel BLN platform operated as an efficient and

377 sensitive platform for fast toxicity screening of various xenobiotics. Moreover, BLN devices

378 indicated broad applicability upon environmental samples, which enabled the direct and in-situ

379 assessment. More importantly, the BLN setup has facilitated a one-step and user-friendly

380 measurement with general analyzer, highlighting its potential for environmental monitoring

381 through a simplified process. Finally, our BLN architecture has exhibited good tolerance during

382 freezing storage as well as great robustness in recycling process, which may endow the BLN

383 system with competitive advantages as a deliverable, ready-to-use device in the large-scale

384 manufacture, even with the potential of being an automatically assembling portable device. 


\section{Figures}
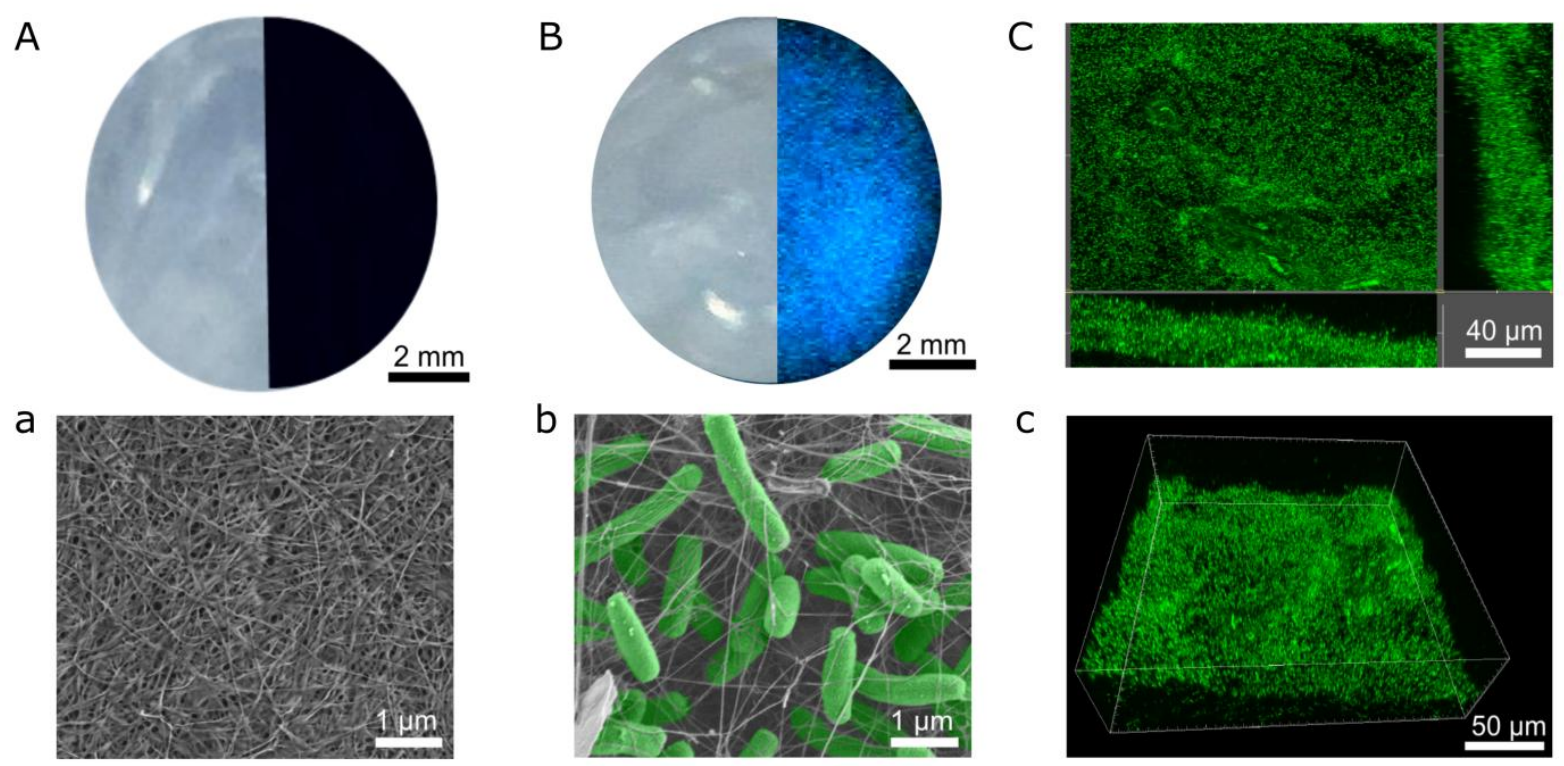

D

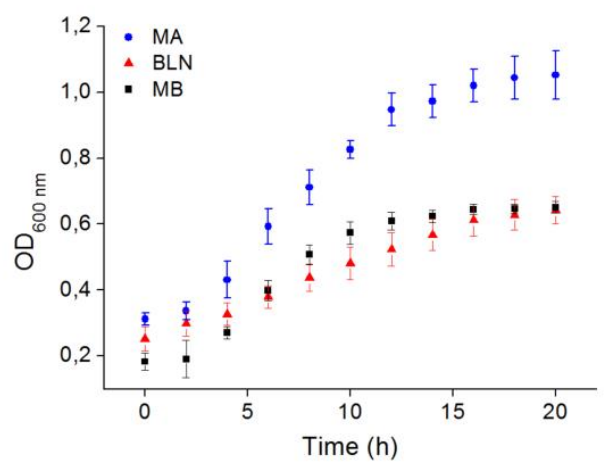

E

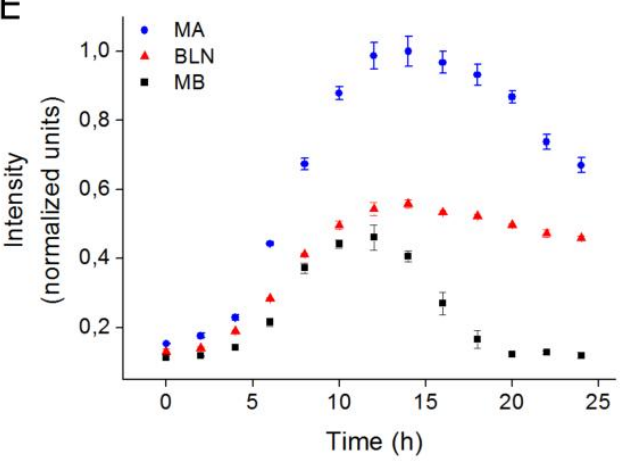

394 Figure 1. The characterization of A. fischeri-decorated BLN platform. (A) Physical appearance

395 of bare nanocellulose by smart phone (left) and its scanned image (right). (a) Scanning electron

396 microscopy (SEM) micrograph of bare bacterial nanocellulose. (B) Physical appearance of $A$.

397 fischeri-decorated BLN by smart phone (left) and its scanned image (right). (b) SEM micrograph 
398 of BLN. (C-c) Confocal microscopy images of BLN. (C) Top view and cross-section. (c) 3d

399 view. (D) The growth tendency. (E) The trend of bioluminescence intensity across time.

400
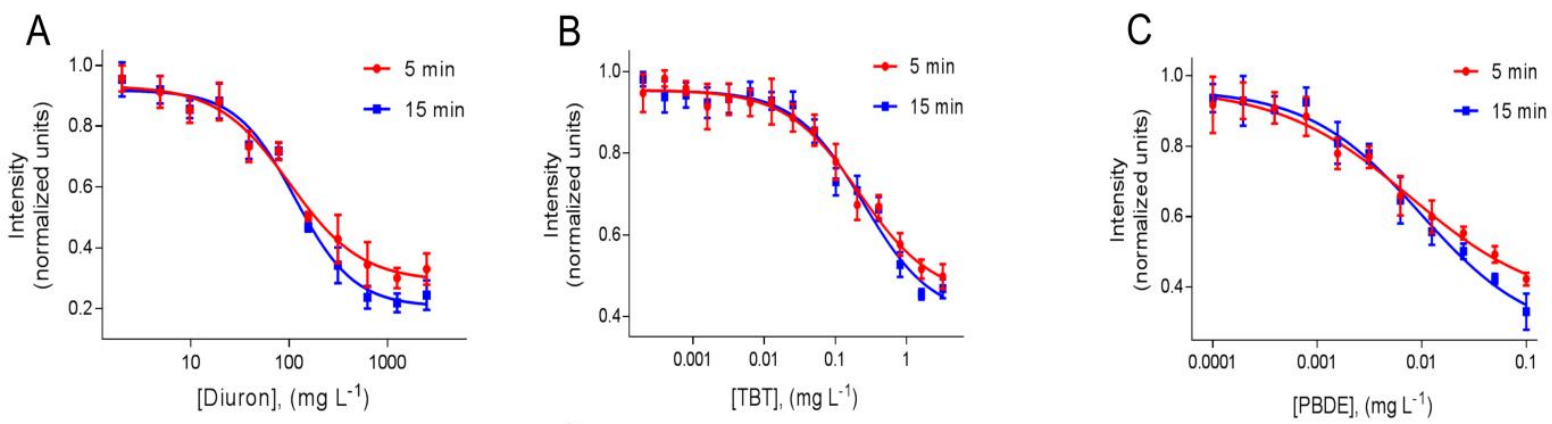

a (min)
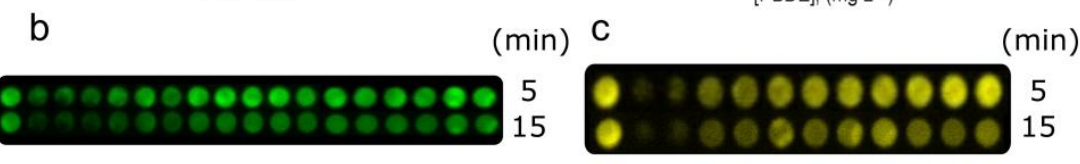

402

Figure 2. The bioluminescence inhibition in A. fischeri-decorated BLN devices via toxic

403 dilutions exposure. (A) The calibration curve related to diuron and (a) the scanned image

404 showing the corresponding bioluminescence. (B) The calibration curve related to TBT and (b)

405 the corresponding scanned image. (C) The calibration curve related to PBDE and (c) the

406 corresponding scanned image. (a-c) Experimental examples. 

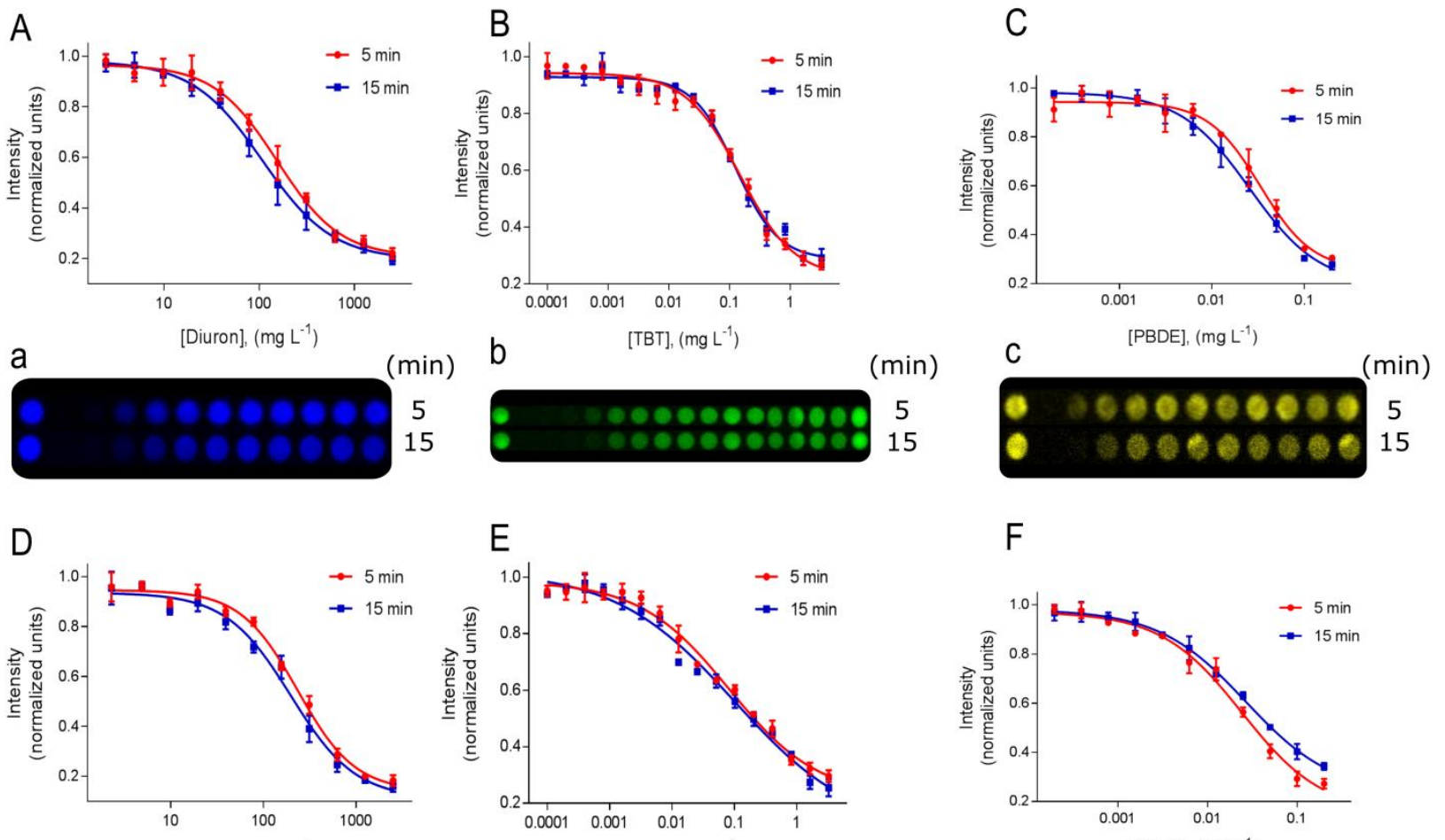

E

$\mathrm{F}$
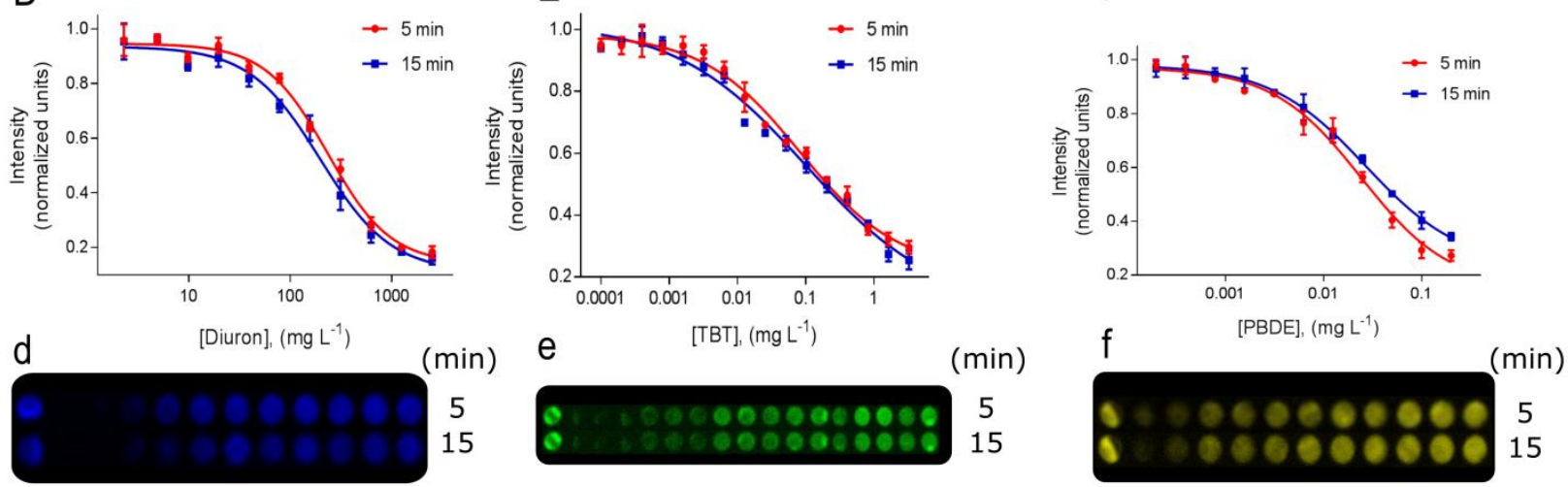

415

416 Figure 3. The bioluminescence inhibition in $A$. fischeri-decorated BLN by toxic-spiked real

417 matrix. (A-C) Analysis in spiked lake water. The calibration curves of (A) diuron, (B) TBT and

418 (C) PBDE and the corresponding scanned images of (a) diuron, (b) TBT and (c) PBDE. (D-F)

419 The calibration curves and (d-f) experimental examples of BLN in spiked seawater. 


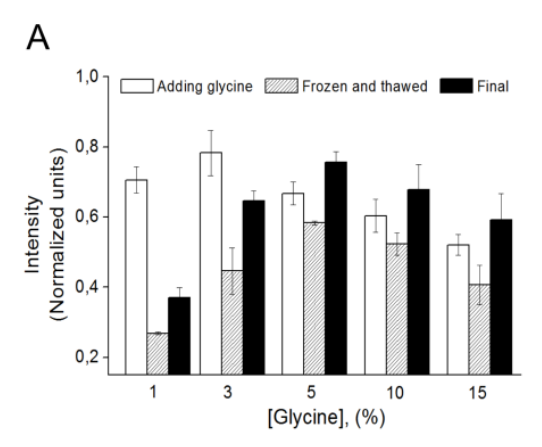

D

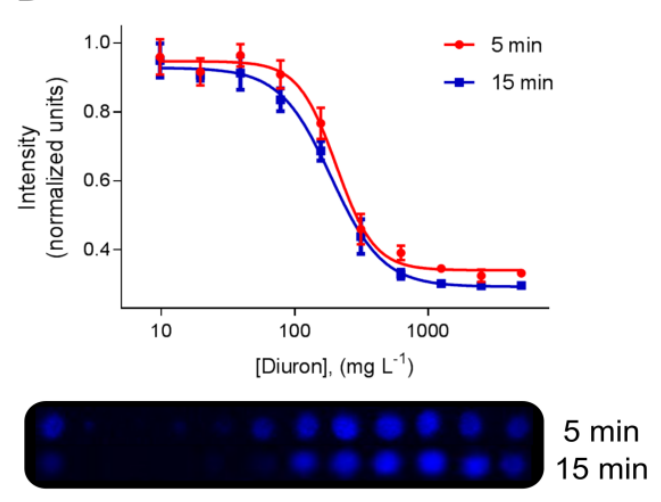

B

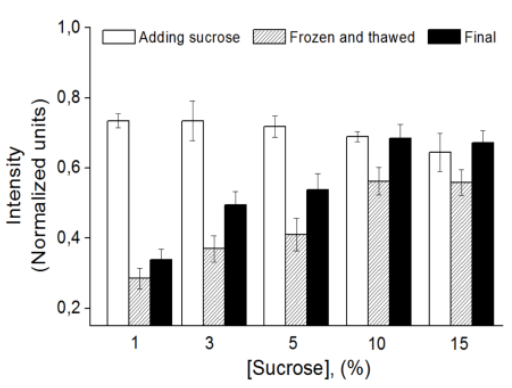

E
C
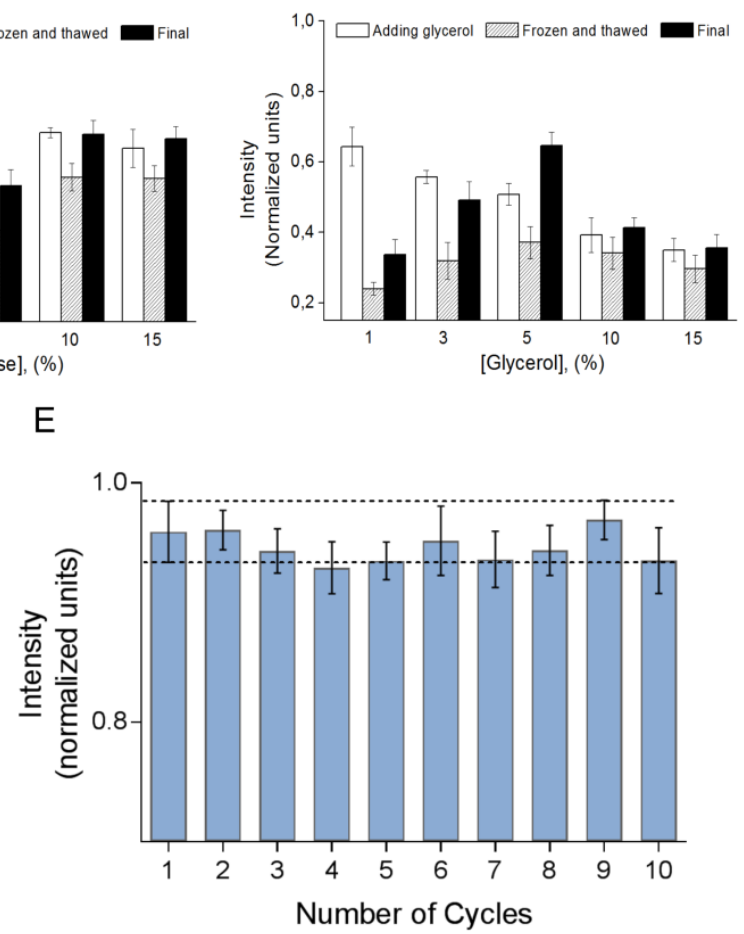

425 Figure 4. BLN after frozen-thawed process. Frozen with (A) glycine; (B) sucrose and (C)

426 glycerol. (D) Bioassay of diuron with the frozen-thawed BLN composites. (E) The

427 bioluminescence of recycled BLN 


\section{Scheme}

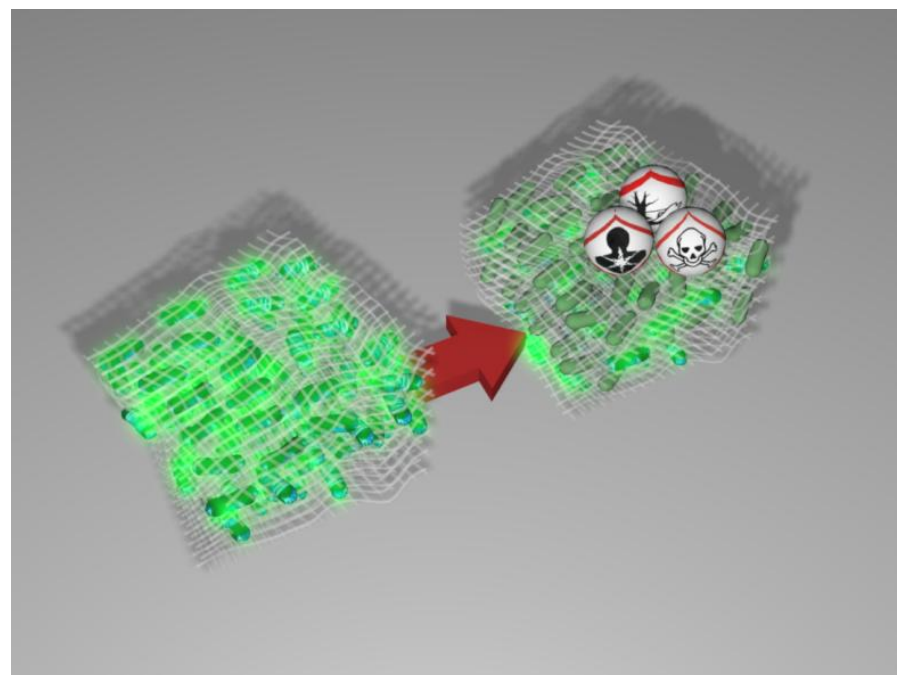

438 Scheme 1. Schematic the proposed nanopaper-based bioassay 


\section{Acknowledgements}

450 This work was supported by the European Commission Program, H2020-WATER, INTCATCH

451 Project (689341). ICN2 acknowledges support from the Severo Ochoa Program (MINECO, 452 Grant SEV-2013-0295). The Nanobiosensors and Bioelectronics Group acknowledges the 453 support from the Generalitat de Cataluña (Grant 2014 SGR 260). Jie Liu acknowledges the 454 support from China Scholarship Council (CSC).

\section{References}

456 [1] Sturm, S.; Hammann, F.; Drewe, J.; Maurer, H. H.; Scholer, A., An automated screening 457 method for drugs and toxic compounds in human serum and urine using liquid chromatography458 tandem mass spectrometry. J. Chromatogr. B 2010, 878 (28), 2726-2732.

459 [2] Maurer, H. H., What is the future of (ultra) high performance liquid chromatography 460 coupled to low and high resolution mass spectrometry for toxicological drug screening? $J$. 461 Chromatogr. A 2013, 1292, 19-24.

462 [3] Blasco, C.; Picó, Y., Prospects for combining chemical and biological methods for 463 integrated environmental assessment. TrAC Trend Anal. Chem. 2009, 28 (6), 745-757.

464 [4] Yu, D.; Liu, J.; Sui, Q.; Wei, Y., Biogas-pH automation control strategy for optimizing 465 organic loading rate of anaerobic membrane bioreactor treating high COD wastewater. 466 Bioresource Technol. 2016, 203, 62-70. 
467 [5] Oller, I.; Malato, S.; Sánchez-Pérez, J. A., Combination of Advanced Oxidation 468 Processes and biological treatments for wastewater decontamination-A review. Sci. Total 469 Environ. 2011, 409 (20), 4141-4166.

470 [6] Parvez, S.; Venkataraman, C.; Mukherji, S., A review on advantages of implementing 471 luminescence inhibition test (Vibrio fischeri) for acute toxicity prediction of chemicals. Environ. 472 Int. 2006, $32(2), 265-268$.

473 [7] Rizzo, L., Bioassays as a tool for evaluating advanced oxidation processes in water and 474 wastewater treatment. Water Res. 2011, 45 (15), 4311-4340.

475 [8] Farré, M.; Barceló, D., Toxicity testing of wastewater and sewage sludge by biosensors, 476 bioassays and chemical analysis. TrAC Trend Anal. Chem. 2003, 22 (5), 299-310.

477 [9] Ma, X. Y.; Wang, X. C.; Ngo, H. H.; Guo, W.; Wu, M. N.; Wang, N., Bioassay based 478 luminescent bacteria: Interferences, improvements, and applications. Sci. Total Environ. 2014, $479468-469,1-11$.

480 [10] Xiao, Y.; Araujo, C. D.; Sze, C. C.; Stuckey, D. C., Toxicity measurement in biological 481 wastewater treatment processes: A review. J. Hazard. Mater. 2015, 286, 15-29.

482 [11] Wieczerzak, M.; Namieśnik, J.; Kudłak, B., Bioassays as one of the Green Chemistry 483 tools for assessing environmental quality: A review. Environ. Int. 2016, 94, 341-361.

484 [12] Hsieh, C.-Y.; Tsai, M.-H.; Ryan, D. K.; Pancorbo, O. C., Toxicity of the 13 priority 485 pollutant metals to Vibrio fisheri in the Microtox ${ }^{\circledR}$ chronic toxicity test. Sci. Total Environ. 2004, $486320(1), 37-50$.

487 [13] Joly, P.; Bonnemoy, F.; Charvy, J.-C.; Bohatier, J.; Mallet, C., Toxicity assessment of the 488 maize herbicides S-metolachlor, benoxacor, mesotrione and nicosulfuron, and their 
489 corresponding commercial formulations, alone and in mixtures, using the Microtox ${ }^{\circledR}$ test.

490 Chemosphere 2013, 93 (10), 2444-2450.

491 [14] Kralj, M. B.; Trebše, P.; Franko, M., Applications of bioanalytical techniques in 492 evaluating advanced oxidation processes in pesticide degradation. TrAC Trend Anal. Chem. 2007, $49326(11), 1020-1031$.

494 [15] Isidori, M.; Lavorgna, M.; Nardelli, A.; Pascarella, L.; Parrella, A., Toxic and genotoxic 495 evaluation of six antibiotics on non-target organisms. Sci. Total Environ. 2005, 346 (1-3), 87-98.

496 [16] van der Grinten, E.; Pikkemaat, M. G.; van den Brandhof, E. -J.; Stroomberg, G. J.; Kraak, 497 M. H. S., Comparing the sensitivity of algal, cyanobacterial and bacterial bioassays to different 498 groups of antibiotics. Chemosphere 2010, 80 (1), 1-6.

499 [17] Journal of Chromatography BHeidari, F.; Asadollahi, M. A.; Jeihanipour, A.; 500 Kheyrandish, M.; Rismani-Yazdi, H.; Karimi, K., Biobutanol production using unhydrolyzed 501 waste acorn as a novel substrate. $R S C A d v$. 2016, 6 (11), 9254-9260.

502 [18] Chang, Z.; Cai, D.; Wang, Y.; Chen, C.; Fu, C.; Wang, G.; Qin, P.; Wang, Z.; Tan, T., 503 Effective multiple stages continuous acetone-butanol-ethanol fermentation by immobilized 504 bioreactors: Making full use of fresh corn stalk. Bioresource Technol. 2016, 205, 82-89.

505 [19] Tang, Y.; Werth, C. J.; Sanford, R. A.; Singh, R.; Michelson, K.; Nobu, M.; Liu, W.-T.; 506 Valocchi, A. J., Immobilization of Selenite via Two Parallel Pathways during In Situ 507 Bioremediation. Environ. Sci. Technol. 2015, 49 (7), 4543-4550.

508 [20] Liu, J.; Chen, S.; Ding, J.; Xiao, Y.; Han, H.; Zhong, G., Sugarcane bagasse as support 509 for immobilization of Bacillus pumilus HZ-2 and its use in bioremediation of mesotrione510 contaminated soils. Appl. Microbiol. Biot. 2015, 99 (24), 10839-10851. 
511 [21] Morales-Narváez, E.; Golmohammadi, H.; Naghdi, T.; Yousefi, H.; Kostiv, U.; Horák, D.;

512 Pourreza, N.; Merkoçi, A., Nanopaper as an Optical Sensing Platform. ACS Nano 2015, 9 (7), $513 \quad 7296-7305$

514 [22] Klemm, D.; Kramer, F.; Moritz, S.; Lindström, T.; Ankerfors, M.; Gray, D.; Dorris, A., 515 Nanocelluloses: A New Family of Nature-Based Materials. Angew. Chem. Int. Edit. 2011, 50 516 (24), 5438-5466.

517 [23] Heli, B.; Morales-Narvaez, E.; Golmohammadi, H.; Ajji, A.; Merkoci, A., Modulation of 518 population density and size of silver nanoparticles embedded in bacterial cellulose via ammonia 519 exposure: visual detection of volatile compounds in a piece of plasmonic nanopaper. Nanoscale $520 \quad 2016,8(15), 7984-7991$.

521 [24] Mertaniemi, H.; Escobedo-Lucea, C.; Sanz-Garcia, A.; Gandía, C.; Mäkitie, A.; Partanen, 522 J.; Ikkala, O.; Yliperttula, M., Human stem cell decorated nanocellulose threads for biomedical 523 applications. Biomaterials 2016, 82, 208-220.

524 [25] Xiong, G.; Luo, H.; Zhu, Y.; Raman, S.; Wan, Y., Creation of macropores in three525 dimensional bacterial cellulose scaffold for potential cancer cell culture. Carbohyd. Polym. 2014, $526 \quad 114,553-557$.

527 [26] Bose, J. L.; Kim, U.; Bartkowski, W.; Gunsalus, R. P.; Overley, A. M.; Lyell, N. L.; 528 Visick, K. L.; Stabb, E. V., Bioluminescence in Vibrio fischeri is controlled by the redox529 responsive regulator ArcA. Mol. Microbiol. 2007, 65 (2), 538-553.

530 [27] de la Escosura-Muñiz, A.; Chunglok, W.; Surareungchai, W.; Merkoçi, A., Nanochannels 531 for diagnostic of thrombin-related diseases in human blood. Biosens. Bioelectro. 2013, 40 (1), $532 \quad 24-31$. 
533 [28] Villa, S.; Vighi, M.; Finizio, A., Experimental and predicted acute toxicity of 534 antibacterial compounds and their mixtures using the luminescent bacterium Vibrio fischeri. 535 Chemosphere 2014, 108, 239-244.

536 [29] Galloway, W. R. J. D.; Hodgkinson, J. T.; Bowden, S. D.; Welch, M.; Spring, D. R.,

537 Quorum Sensing in Gram-Negative Bacteria: Small-Molecule Modulation of AHL and AI-2 538 Quorum Sensing Pathways. Chem. Rev. 2011, 111 (1), 28-67.

539 [30] Ng, W.-L.; Bassler, B. L., Bacterial Quorum-Sensing Network Architectures. Annu. Rev. $540 \quad$ Genet. 2009, 43, 197-222.

541 [31] L. Cacicedo, M.; E. León, I.; S. Gonzalez, J.; M. Porto, L.; A. Alvarez, V.; Castro, G. R.,

542 Modified bacterial cellulose scaffolds for localized doxorubicin release in human colorectal HT54329 cells. Colloid. Surface. B 2016, 140, 421-429.

544 [32] Ah S SensFavi, P. M.; Ospina, S. P.; Kachole, M.; Gao, M.; Atehortua, L.; Webster, T. J., 545 Preparation and characterization of biodegradable nano hydroxyapatite-bacterial cellulose 546 composites with well-defined honeycomb pore arrays for bone tissue engineering applications. 547 Cellulose 2016, 23 (2), 1263-1282.

548 [33] Svensson, A.; Nicklasson, E.; Harrah, T.; Panilaitis, B.; Kaplan, D. L.; Brittberg, M.; 549 Gatenholm, P., Bacterial cellulose as a potential scaffold for tissue engineering of cartilage. $550 \quad$ Biomaterials 2005, $26(4), 419-431$.

551 [34] Stebbing, A. R. D., Hormesis - The stimulation of growth by low levels of inhibitors. 552 Sci. Total Environ. 1982, 22 (3), 213-234.

553 [35] Calabrese, E. J.; Baldwin, L. A., Hormesis: the dose-response revolution. Annu. Rev. 554 Pharmacol.Toxicol. 2003, 43 (1), 175-197. 
555 [36] Donnez, J.; Martinez-Madrid, B.; Jadoul, P.; Van Langendonckt, A.; Demylle, D.;

556 Dolmans, M.-M., Ovarian tissue cryopreservation and transplantation: a review. Hum. Reprod.

557 Update 2006, 12 (5), 519-535.

558 [37] Kovalevsky, G.; Carney, S. M.; Morrison, L. S.; Boylan, C. F.; Neithardt, A. B.; Feinberg,

559 R. F., Should embryos developing to blastocysts on day 7 be cryopreserved and transferred:

560 an analysis of pregnancy and implantation rates. Fertil. Steril. 2013, 100 (4), 1008-1012.

561 [38] Subbarayan, K.; Rolletschek, H.; Senula, A.; Ulagappan, K.; Hajirezaei, M.-R.; Keller, E.

562 R. J., Influence of oxygen deficiency and the role of specific amino acids in cryopreservation of 563 garlic shoot tips. BMC Biotechnol. 2015, $15(1), 40$. 